2021-03-15

\title{
Nonlinear Trajectory Tracking Control for Winged eVTOL UAVs
}

Jacob Willis

jbwillis272@gmail.com

Randal W. Beard

beard@byu.edu

Follow this and additional works at: https://scholarsarchive.byu.edu/facpub

Part of the Electrical and Computer Engineering Commons

\section{BYU ScholarsArchive Citation}

Willis, Jacob and Beard, Randal W., "Nonlinear Trajectory Tracking Control for Winged eVTOL UAVs" (2021). Faculty Publications. 5011.

https://scholarsarchive.byu.edu/facpub/5011

This Conference Paper is brought to you for free and open access by BYU ScholarsArchive. It has been accepted for inclusion in Faculty Publications by an authorized administrator of BYU ScholarsArchive. For more information, please contact ellen_amatangelo@byu.edu. 


\title{
Nonlinear Trajectory Tracking Control for Winged eVTOL UAVs
}

\author{
Jacob B. Willis* and Randal W. Beard**
}

\begin{abstract}
Current control methods for winged eVTOL UAVs consider the vehicle primarily as a fixed-wing aircraft with the addition of vertical thrust used only during takeoff and landing. These methods provide good long-range flight handling but fail to consider the full dynamics of the vehicle for tracking complex trajectories. We present a trajectory tracking controller for the full dynamics of a winged eVTOL UAV in hover, fixed-wing, and partially transitioned flight scenarios. We show that in lowto moderate-speed flight, trajectory tracking can be achieved using a variety of pitch angles. In these conditions, the pitch of the vehicle is a free variable which we use to minimize the necessary thrust, and therefore energy consumption, of the vehicle. We use a geometric attitude controller and an airspeeddependent control allocation scheme to operate the vehicle at a wide range of airspeeds, flight path angles, and angles of attack. We provide simulation results and theoretical guarantees for the stability of the proposed control scheme assuming a standard aerodynamic model.
\end{abstract}

\section{INTRODUCTION}

Winged electric vertical takeoff and landing (eVTOL) unmanned aerial vehicles (UAVs) are capable of vertical flight as well as sustained horizontal flight using lifting surfaces. These complementary features make them ideal in a variety of applications where endurance and maneuverability are both required. Current control methods provide good longrange flight handling but fail to consider the full dynamics of the vehicle for tracking complex trajectories. It is during the phase of flight immediately after takeoff and before landing that the vehicle is most likely to encounter obstacles and need to navigate in a precise manner. While agility is important during these maneuvers, efficiency also remains an important objective. Here we present a controller which is capable of controlling a winged eVTOL in hover, fixed-wing, and partially transitioned flight scenarios. The control scheme is generally applicable to a variety of winged eVTOL UAVs including vectored thrust and lift-cruise vehicles 1

Trajectory tracking for multirotor UAVs has been extensively studied and provides a foundation to the work we present here. Geometric control [1] provides excellent tracking performance of continuous position and yaw trajectories and is frequently used for multirotor control [2], [3], [4]. This method takes advantage of the differentially flat [4] and underactuated dynamics of multirotors to map

\footnotetext{
${ }^{*}$ Graduate research assistant, BYU MAGICC lab.

** Professor of Electrical and Computer Engineering, Brigham Young University, beardebyu . edu

***This work has been funded by the Center for Unmanned Aircraft Systems (C-UAS), a National Science Foundation Industry/University Cooperative Research Center (I/UCRC) under NSF award No. IIP-1650547, along with significant contributions from C-UAS industry members.

${ }^{1}$ See https: / / evtol. news/classifications
}

from a three-times differentiable position trajectory and onetime differentiable yaw trajectory to feed-forward thrust and attitude commands. Proportional-derivative feedback control causes the vehicle to converge asymptotically to the desired trajectory.

While multirotor UAVs have excellent maneuverability, they lack the ability to perform long-endurance flights. Many approaches to addressing this drawback exist, including adding extra degrees of freedom to multirotors [5], [6] or creating winged eVTOL UAVs which hybridize the use of thrust for vertical takeoff and landing and the use of wings for long-endurance flight [7]. Winged eVTOLs provide the largest opportunity for improvements in endurance over multirotor and other eVTOL UAVs, and promise more maneuverable flight than fixed-wing vehicles. These benefits come, however, at the cost of increased complexity over multirotor and fixed-wing vehicles [8]. During maneuvers which transition between VTOL and fixed-wing flight, a winged eVTOL experiences high-angle-of-attack flight where stall and unsteady aerodynamics have a large impact on the behavior of the vehicle.

Most proposed control methods for winged eVTOLs differ with respect to how the transition is controlled. Gain scheduling using empirical data [9] and incremental nonlinear dynamic inversion [10] have been used to perform stability control for human-piloted winged eVTOLs. The popular PX4 flight controller [11] mixes control inputs from a multirotor and a fixed-wing controller over a finite transition period ${ }^{2}$ This method allows the use of the vehicle in hover and fixed-wing configurations but does not allow the use of intermediate flight modes. Notarstefano and Hauser [12] investigate the equilibrium manifold of a VTOL aircraft with a gimballed tilt-rotor having two degrees of freedom. Their investigation assumes a constant rotor attitude and coordinated turn flight, allowing them to avoid modelling the aerodynamic coefficients of the vehicle. A nonlinear controller for the hover-to-fixed-wing transition of a quad tiltrotor is developed in [13]. The authors assume the vehicle remains at zero pitch and zero angle-of-attack, conditions which limit the transition maneuver to be at constant altitude and along a straight line. Nonlinear trajectory tracking control of a quad tilt-rotor is presented by Anglade, et al. [14]. A simplified aerodynamic model is used to enable stability proofs. The vehicle always points in the direction of travel, an assumption which aids efficiency in forward flight, but fails for purely vertical, sideways, and rearward flight.

${ }^{2}$ For more details, see https://dev.px4.io/master/
en/flight_stack/controller_diagrams.html\#
vtol-flight-controller
vtol-flight-controller 
This significantly reduces the number of possible maneuvers the vehicle can make, reducing the advantage of a highly maneuverable airframe. Differential flatness for a tilt-wing eVTOL based on Euler angles and using position, angleof-attack, and sideslip as flat outputs is shown by [15]. A velocity controller for a tilt-wing UAV is developed in [16]. The nested rate controller uses virtual outputs which are converted to the proper actuator commands using an allocation scheme which varies with the current flight state.

The approach we take is to track a trajectory consisting of the three-dimensional position and the yaw of the vehicle, similar to what is done for multirotors in [1]. By including the yaw angle in the trajectory, we are able to command a much larger set of flight behaviors than is possible when pointing the nose of the vehicle along the velocity vector as done in [14]. The consequence of this is that efficient flight, where the vehicle points in the direction of its velocity, becomes a planning problem. While we don't prove differential flatness for a winged eVTOL, our approach to control design follows a similar progression as a differential flatness proof. It would be trivial to show that our model is differentially flat with position, yaw, and pitch or thrust angle as flat outputs by following our trajectory tracking control derivation without feedback terms.

In section II we present the winged eVTOL model as a generic rigid body capable of producing forces in its longitudinal plane. Our controller development begins in section III where we initially assume zero pitch and choose the roll angle such that the desired acceleration vector resides in the body longitudinal plane. We then find the optimal pitch angle using a nonlinear optimization of the longitudinal aerodynamics as shown in section IV. This augments the desired attitude, which, as described in section $\mathrm{V}$, is controlled using a nonlinear attitude controller and proportional-integral-derivative (PID) loops outputting virtual torque commands. The virtual torques and thrust are then allocated to the appropriate actuators using a linear control allocation in section VI. A flowchart showing these control stages and the control variables passed between them is shown in fig. 1 1 . The controller is simulated for a tiltrotor UAV in our Python3 simulation environment described in [17]. While we apply the control scheme to a tilt-rotor, it is generally applicable to winged eVTOLs which produce thrust in the body longitudinal plane. The platform-specific aspects of the control design are in the constraints of the pitch optimization and in the control allocation scheme.

\section{DYNAMiC MODEL}

Throughout this paper the notation $\boldsymbol{r}_{a / b}^{c}$ is used to denote a vector quantity $\boldsymbol{r}$ of frame $a$ with respect to frame $b$ and expressed in frame $c$. We use $i$ to refer to the north-east-down inertial frame, and $b$ to refer to the true body-fixed frame. The reference, desired, desired pitch, and control frames, described when they are used, are denoted $r, d, p$, and $c$ respectively.

Let $\boldsymbol{p}_{b / i}^{i} \in \mathbb{R}^{3}$ be the position of the vehicle body with

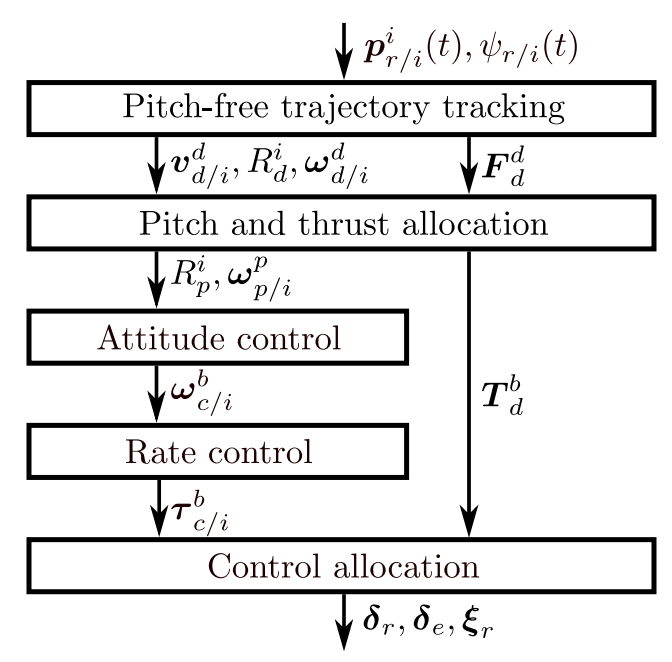

Fig. 1. The flow of the control variables through the various stages described in the paper. At the top-level, a three-times differentiable position and a differentiable yaw trajectory is provided to the controller. The trajectory tracking controller assumes zero pitch and finds the desired attitude, rates, and applied forces such that all of the necessary applied forces reside in the body longitudinal plane. The pitch of the vehicle is then optimized to minimize the necessary thrust. The geometric attitude controller assumes instantaneous angular rates which are controlled by highrate PID loops. The virtual torque and thrust outputs are converted to rotor thrust, elevon, and rotor angle commands using a linear control allocation scheme.

respect to the inertial frame, $\boldsymbol{v}_{b / i}^{i} \in \mathbb{R}^{3}$ be the velocity of the vehicle body with respect to the inertial frame, $R_{b}^{i}=$ $\left[\boldsymbol{x}_{b}^{i}, \boldsymbol{y}_{b}^{i}, \boldsymbol{z}_{b}^{i}\right] \in \mathrm{SO}(3)$ be the rotation matrix which rotates body-frame vectors into the inertial frame, with its columns $\boldsymbol{x}_{b}^{i}, \boldsymbol{y}_{b}^{i}$, and $\boldsymbol{z}_{b}^{i}$ respectively the body-frame $\boldsymbol{x}, \boldsymbol{y}$, and $\boldsymbol{z}$ basis vectors represented in the inertial frame, $\boldsymbol{\omega}_{b / i}^{b} \in \mathbb{R}^{3}$ be the angular velocity of the vehicle body with respect to the inertial frame and expressed in the body frame, $m$ be the mass of the vehicle, $J \in \mathbb{R}^{3 \times 3}$ be the inertia matrix, and $g$ be the acceleration due to gravity. We define the operator which forms a $3 \times 3$ skew-symmetric matrix from a vector $\boldsymbol{\omega} \in \mathbb{R}^{3}$ as $\lfloor\boldsymbol{\omega}\rfloor_{\times}$, the canonical unit vectors in $\mathbb{R}^{3}$ as $\boldsymbol{e}_{1}, \boldsymbol{e}_{2}$, and $\boldsymbol{e}_{3}$, and the zero vector as $\mathbf{0}$. For control design, we assume that the vehicle is yaw-stable and that sideways flight occurs only at low airspeed, so forces in the lateral plane of the vehicle are negligible. The rigid body dynamics are then given by

$$
\begin{aligned}
\dot{\boldsymbol{p}}_{b / i}^{i} & =\boldsymbol{v}_{b / i}^{i} \\
\dot{\boldsymbol{v}}_{b / i}^{i} & =g \boldsymbol{e}_{3}+\frac{1}{m} R_{b}^{i} B \boldsymbol{F}_{b}^{b} \\
\dot{R}_{b}^{i} & =R_{b}^{i}\left\lfloor\boldsymbol{\omega}_{b / i}^{b}\right\rfloor_{\times} \\
\dot{\boldsymbol{\omega}}_{b / i}^{b} & =-J^{-1}\left\lfloor\boldsymbol{\omega}_{b / i}^{b}\right\rfloor_{\times} J \boldsymbol{\omega}_{b / i}^{b}+J^{-1} \boldsymbol{M}_{b}^{b}
\end{aligned}
$$

where $\boldsymbol{F}_{b}^{b}=\left[F_{x, b}^{b}, F_{z, b}^{b}\right]^{\top}$ is the vector of applied forces in the body longitudinal plane,

$$
B=\left[\begin{array}{ll}
1 & 0 \\
0 & 0 \\
0 & 1
\end{array}\right]
$$


expands $\boldsymbol{F}$ from two dimensions to three, and $\boldsymbol{M}_{b}^{b}=$ $\left[M_{x, b}^{b}, M_{y, b}^{b}, M_{z, b}^{b}\right]^{\top}$ is the vector of applied moments in the body frame.

The full aerodynamic models for $\boldsymbol{F}$ and $\boldsymbol{M}$ are given in [17] and omitted here for brevity. The simulation model described in [17] and used for the results in section VII extends eq. (1) to include lateral aerodynamic forces. Portions of the aerodynamic models will be given as needed in subsequent sections.

\section{Pitch-Free Geometric Trajectory Tracking}

From the model in eq. (1) it is apparent that a winged eVTOL can be generally considered as a rigid body capable of producing moments about its three body axes as well as producing a force in its body longitudinal plane. The forces produced in the body longitudinal plane are the sum of thrust and aerodynamic forces. The aerodynamic forces the wing generates are determined by the airspeed and the angle-ofattack. Because the thrust has two degrees of freedom which can be directly controlled, and the pitch affects the amount of aerodynamic forces the vehicle produces, the mapping from pitch and thrust to total body forces is many-to-one. We therefore initially assume zero pitch and find the desired total body forces. Then in section IV the pitch and thrust are determined.

\section{A. Pitch-Free Geometric Control}

We will first focus on trajectory tracking, finding a desired attitude $R_{d}^{i}$ that will orient the desired force $\boldsymbol{F}_{d}^{d}$ to move the vehicle towards a position and yaw reference trajectory. We begin by ignoring thrust angle constraints, so $\boldsymbol{F}_{d}^{d}$ can be any vector in the longitudinal plane; thrust angle constraints are handled by the optimization in section IV. The position and yaw reference trajectories are $\boldsymbol{p}_{r / i}^{i}(t)$, and $\psi_{r / i}(t)$ respectively, where the subscript $r$ denotes the reference frame. We assume that $\boldsymbol{p}_{r / i}^{i}(t)$ is three times differentiable and that $\psi_{r / i}^{i}(t)$ is two times differentiable.

We start by defining the position error

$$
\boldsymbol{p}_{b / r}^{i}=\boldsymbol{p}_{b / i}^{i}-\boldsymbol{p}_{r / i}^{i}
$$

Differentiating, the velocity and acceleration errors give the error-state model

$$
\begin{aligned}
& \dot{\boldsymbol{p}}_{b / r}^{i}=\boldsymbol{v}_{b / i}^{i}-\dot{\boldsymbol{p}}_{r / i}^{i} \\
& \ddot{\boldsymbol{p}}_{b / r}^{i}=\dot{\boldsymbol{v}}_{b / i}^{i}-\ddot{\boldsymbol{p}}_{r / i}^{i}=g \boldsymbol{e}_{3}+\frac{1}{m} R_{b}^{i} B \boldsymbol{F}_{b}^{b}-\ddot{\boldsymbol{p}}_{r / i}^{i} .
\end{aligned}
$$

We now find the desired attitude and force vector that will cause the tracking error $\left\|\boldsymbol{p}_{b / r}\right\|$ to go to zero.

Theorem 1. Given the dynamics listed in Equation (4)-(5), if the desired force is

$$
\boldsymbol{F}_{d}^{d}=\left[\begin{array}{l}
\left(\boldsymbol{x}_{d}^{i}\right)^{\top} \boldsymbol{f}_{d}^{i} \\
\left(\boldsymbol{z}_{d}^{i}\right)^{\top} \boldsymbol{f}_{d}^{i}
\end{array}\right]
$$

and the desired attitude is

$$
R_{d}^{i}=\left[\boldsymbol{x}_{d}^{i}, \boldsymbol{y}_{d}^{i}, \boldsymbol{z}_{d}^{i}\right]
$$

where

$$
\begin{aligned}
\boldsymbol{f}_{d}^{i} & =m\left(\ddot{\boldsymbol{p}}_{r / i}^{i}-g \boldsymbol{e}_{3}-K_{p} \boldsymbol{p}_{b / r}^{i}-K_{d} \dot{\boldsymbol{p}}_{b / r}^{i}\right) \\
\boldsymbol{x}_{d}^{i} & =\left[\cos \psi_{r / i}, \sin \psi_{r / i}, 0\right]^{\top}, \\
\boldsymbol{y}_{d}^{i} & =\frac{\boldsymbol{x}_{d}^{i} \times \boldsymbol{f}_{d}^{i}}{\left\|\boldsymbol{x}_{d}^{i} \times \boldsymbol{f}_{d}^{i}\right\|} \\
\boldsymbol{z}_{d}^{i} & =\boldsymbol{x}_{d}^{i} \times \boldsymbol{y}_{d}^{i},
\end{aligned}
$$

and where $K_{p}=K_{p}^{\top}>0$ and $K_{d}=K_{d}^{\top}>0$, then $\left\|\dot{\boldsymbol{p}}_{b / r}^{i}\right\| \rightarrow 0$ and $\left\|\boldsymbol{p}_{b / r}^{i}\right\| \rightarrow 0$.

The proof is omitted for brevity but consists of showing that the selection of $\boldsymbol{F}_{d}^{d}$ and $R_{d}^{i}$ implies that

$$
\ddot{\boldsymbol{p}}_{b / r}^{i}+K_{d} \dot{\boldsymbol{p}}_{b / r}^{i}+K_{p} \boldsymbol{p}_{b / r}^{i}=0 .
$$

\section{B. Feed-Forward Angular Rates}

The rate of change of the desired attitude of the vehicle provides a feed-forward term for the commanded angular rates of the vehicle. The feed-forward angular rates $\boldsymbol{\omega}_{d / i}^{d}$ can be determined analytically by differentiating eqs. (9) to (11). Substituting $R_{d}^{i}$ for $R_{b}^{i}$ and $\boldsymbol{\omega}_{d / i}^{d}$ for $\boldsymbol{\omega}_{b / i}^{d}$ in eq. (1c) and solving for $\boldsymbol{\omega}_{d / i}^{d}$ by multiplying on the left by $R_{d}^{i}$ and using the inverse skew operator, $(\cdot)^{\vee}$, we have

$$
\boldsymbol{\omega}_{d / i}^{d}=\left(R_{d}^{i^{\top}} \dot{R}_{d}^{i}\right)^{\vee}
$$

The time derivative of $R_{d}^{i}$ can be found by taking the time derivative of $\boldsymbol{x}_{d}^{i}, \boldsymbol{y}_{d}^{i}$, and $\boldsymbol{z}_{d}^{i}$, as shown in appendix $\mathrm{B}$.

\section{Pitch And Thrust Optimization}

By assuming zero pitch, the trajectory tracking controller is able to satisfy the vehicle's dynamic constraints. The task now remains to find the optimal pitch to achieve the desired applied forces, $\boldsymbol{F}_{d}^{d}$, found in section III To do so, we find the pitch angle which minimizes the thrust necessary to achieve $\boldsymbol{F}_{d}^{d}$. We begin by describing the relationship between the total forces $\boldsymbol{F}_{d}^{d}$, the thrust $\boldsymbol{T}_{d}^{p}$ and the aerodynamic forces $\boldsymbol{F}_{\text {aero. }}$. We let $p$ denote the desired pitch frame, which is rotated about the $\boldsymbol{y}_{d}^{d}$ axis from the desired frame by $\theta_{p / d}$.

We assume zero wind so we let $V_{a}=\left\|\boldsymbol{v}_{d / i}^{d}\right\|$ be the magnitude of the desired airspeed. We define the angle-ofattack to be

$$
\alpha_{p / d}=\tan ^{-1}\left(\frac{\boldsymbol{v}_{z, d / i}^{p}}{\boldsymbol{v}_{x, d / i}^{p}}\right) .
$$

The difference between the angle-of-attack and the pitch angle is the flight path angle,

$$
\gamma_{p / d}=\theta_{p / d}-\alpha_{p / d}=\tan ^{-1}\left(\frac{-\boldsymbol{v}_{z, d / i}^{d}}{\boldsymbol{v}_{x, d / i}^{d}}\right) .
$$

We then define the longitudinal aerodynamic force vector to be

$$
\boldsymbol{F}_{\text {aero }}\left(\alpha, V_{a}\right)=\left[\begin{array}{cc}
\cos \alpha & -\sin \alpha \\
\sin \alpha & \cos \alpha
\end{array}\right]\left[\begin{array}{l}
-F_{\text {drag }}\left(\alpha, V_{a}\right) \\
-F_{\text {lift }}\left(\alpha, V_{a}\right)
\end{array}\right]
$$


where $F_{\text {lift }}$ and $F_{\text {drag }}$ are defined in [17]. We define the angle of the thrust with respect to the desired frame as

$$
\xi_{T / p}=\tan ^{-1}\left(\frac{-\boldsymbol{T}_{z, d}^{p}}{\boldsymbol{T}_{x, d}^{p}}\right) .
$$

The relationship between the aerodynamic forces, thrust, and total forces is then

$$
\boldsymbol{F}_{\text {aero }}\left(\alpha_{p / d}, V_{a}\right)+\boldsymbol{T}_{d}^{p}=\bar{R}_{d}^{p} \boldsymbol{F}_{d}^{d}
$$

where

$$
\bar{R}_{d}^{p}=\left[\begin{array}{cc}
\cos \theta_{p / d} & -\sin \theta_{p / d} \\
\sin \theta_{p / d} & \cos \theta_{p / d}
\end{array}\right] .
$$

These quantities are depicted in fig. 2 .

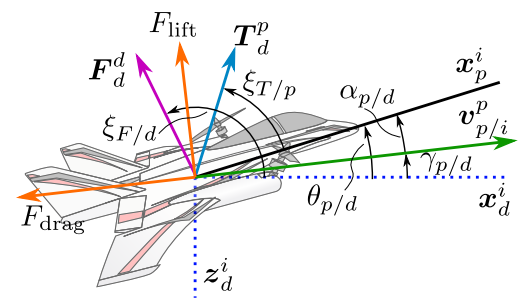

Fig. 2. Depiction of the vectors and angles used in computing the aerodynamic forces and thrust. The origin of the pitch frame coincides with the origin of the desired frame. The $\boldsymbol{x}_{d}^{i}$ axis of the desired frame is in the horizontal plane, that is, it is perpendicular to the $\boldsymbol{z}_{i}^{i}$ axis of the inertial frame.

\section{A. Pitch and Thrust Optimization}

The optimization variables are the desired thrust $\boldsymbol{T}_{d}^{p}$, and the desired pitch angle $\theta_{p / d}$. To use the most efficient travel mode possible the optimization objective is to minimize $\left\|\boldsymbol{T}_{d}^{p}\right\|$. Constraints ensure $\boldsymbol{F}_{d}^{d}$ is achieved within limits on the pitch and thrust angle. We additionally enforce a constraint on the rate of change of the pitch from the previous optimal pitch to ensure dynamically feasible results.

The optimization is then

$$
\begin{array}{ll}
\text { given } & V_{a}, \gamma_{p / d}, \boldsymbol{F}_{d}^{d} \\
\text { minimize } & \left\|\boldsymbol{T}_{d}^{p}\right\| \\
\text { with respect to } & \boldsymbol{T}_{d}^{p}, \theta_{p / d} \\
\text { subject to } & \boldsymbol{F}_{\text {aero }}\left(\theta_{p / d}-\gamma_{p / d}, V_{a}\right)+\boldsymbol{T}_{d}^{p}=\bar{R}_{d}^{p} \boldsymbol{F}_{d}^{d} \\
& \theta_{\min } \leq \theta_{p / d} \leq \theta_{\max } \\
& \xi_{\min } \leq \xi_{T / p} \leq \xi_{\max } \\
& \left|\theta_{p / d}-\theta_{\text {prev }}\right| \leq \Delta t \omega_{y_{\max }}
\end{array}
$$

with $\gamma_{p / d}$ held constant. We solve the optimization using the SNOPT optimizer [18] and the PyOptSparse library [19].

As given in eq. 20), the optimization will fail when the desired force vector is outside the range that is achievable while satisfying $\theta_{\min } \leq \theta_{p / d} \leq \theta_{\max }$ and $\xi_{\min } \leq \xi_{T / p} \leq$ $\xi_{\max }$. Some of these scenarios are dynamically infeasible, and should be avoided by the trajectory planner. But when the desired force vector points above and to the rear of the vehicle, such as would occur when the vehicle is rapidly decelerating or flying backwards, relaxing the maximum pitch constraint allows the vehicle to pitch up higher and produce the desired force vector. To accommodate this scenario, we define

$$
\xi_{F / d}=\tan ^{-1}\left(\frac{-\boldsymbol{F}_{z, d}^{d}}{\boldsymbol{F}_{x, d}^{d}}\right)
$$

and we modify the constraint on $\theta_{p / d}$ to be

$$
\theta_{\min } \leq \theta_{p / d} \leq \theta_{\max }^{\star}
$$

where $\theta_{\max }^{\star}=\max \left(\theta_{\max }, \xi_{F / d}-90^{\circ}\right)$.

\section{B. Augmenting with optimal thrust and pitch}

Once the optimal thrust and pitch have been found, it is necessary to augment the desired vehicle attitude to include pitch, and to represent the angular rates and thrust in the appropriate reference frames. To do so, we use the angleaxis representation to find a rotation matrix which rotates vectors about the $\boldsymbol{y}_{d}^{d}$ axis by $\theta_{p / d}$, and multiply it by $R_{d}^{i}$ on the left. Since $\boldsymbol{y}_{d}^{d}=\boldsymbol{e}_{2}$, we have

$$
R_{p}^{d}=\exp \left(\theta_{p / d}\left\lfloor\boldsymbol{e}_{2}\right\rfloor_{\times}\right),
$$

and $R_{p}^{i}=R_{d}^{i} R_{p}^{d}$. We rotate the desired angular rates into the pitched frame

$$
\boldsymbol{\omega}_{p / i}^{p}=\left(R_{p}^{d}\right)^{T} \boldsymbol{\omega}_{d / i}^{d}
$$

and we rotate the optimal thrust into the vehicle's body frame

$$
\boldsymbol{T}_{d}^{b}=B^{T} R_{d}^{b} R_{p}^{d} B \boldsymbol{T}_{d}^{p} .
$$

\section{Attitude And Angular Rate Control}

The pitch-augmented rotation matrix $R_{p}^{i}$ represents the attitude that will cause the vehicle to most efficiently converge to the reference trajectory. With a combination of differential thrust and the use of the elevons, we are able to produce torques about the three body axes to achieve the desired attitude. To allow for eventual high-update-rate control of the angular rates on an embedded flight controller, we separate the attitude and rate control into two stages.

\section{A. Attitude Control}

Here we are concerned with determining the necessary angular rates to achieve and track the desired attitude. Assuming that the body angular rates $\boldsymbol{\omega}_{b / i}^{b}$ converge instantaneously to the commanded angular rates $\boldsymbol{\omega}_{c / i}^{b}$, the attitude dynamics, from eq. (1), are

$$
\dot{R}_{b}^{i}=R_{b}^{i}\left\lfloor\omega_{c / i}^{b}\right\rfloor_{\times} .
$$

Theorem 2. Let $\mathbb{P}_{a}(R)=\frac{1}{2}\left(R-R^{T}\right)$ be the operator which takes the antisymmetric part of $R$, and let $K_{R} \in \mathbb{R}^{3 \times 3}$ be a symmetric, positive definite gain matrix. With $R_{p}^{b}=$ $\left(R_{b}^{i}\right)^{T} R_{p}^{i}$, and supposing that for all time $\frac{1}{2} \operatorname{tr}\left(I-R_{p}^{b}\right)<2$, the commanded angular velocity

$$
\boldsymbol{\omega}_{c / i}^{b}=R_{p}^{b} \boldsymbol{\omega}_{p / i}^{p}+K_{R} \mathbb{P}_{a}\left(R_{p}^{b}\right)^{\vee}
$$

will cause $R_{b}^{i} \rightarrow R_{p}^{i}$ and $\boldsymbol{\omega}_{b / i}^{b} \rightarrow \boldsymbol{\omega}_{p / i}^{b}$ asymptotically.

The proof of theorem 2 is not given due to space constraints, but is similar to the proof in [1]. 


\section{B. Rate Control}

Given $\boldsymbol{\omega}_{c / i}^{b}$ from eq. 27, we use high-update-rate PID loops as defined in theorem 3 to control the angular rate dynamics.

Theorem 3. Let $K_{\omega, p}, K_{\omega, d}, K_{\omega, i} \in \mathbb{R}^{3 \times 3}$ be diagonal matrices with positive entries, and define $\boldsymbol{\omega}_{c / b}^{b}=\boldsymbol{\omega}_{c / i}^{b}-\boldsymbol{\omega}_{b / i}^{b}$. Then

$$
\boldsymbol{\tau}_{c / b}^{b}=K_{\omega, p} \boldsymbol{\omega}_{c / b}^{b}+K_{\omega, d} \dot{\boldsymbol{\omega}}_{c / b}^{b}+K_{\omega, i} \int_{0}^{t} \boldsymbol{\omega}_{c / b}^{b} d t
$$

will cause $\boldsymbol{\omega}_{b / i}^{b} \rightarrow \boldsymbol{\omega}_{c / i}^{b}$.

\section{Low-LeVel Control Allocation}

While the previous sections assume a fairly general model for a winged eVTOL UAV, this section develops the bridge between the general model and the real vehicle. Here the goal is to transform the desired thrust $\boldsymbol{T}_{d}^{b}$ and the desired torque $\boldsymbol{\tau}_{c / b}^{b}$ to individual motor throttle, motor angle, and control surface commands. In appendix C we derive an allocation matrix $\mathbf{G}$ which provides a linear relationship between the thrust and torque, and a vector consisting of rotor thrust components $\boldsymbol{Z}$ and elevon commands $\boldsymbol{\delta}_{e}$,

$$
\left[\begin{array}{c}
\boldsymbol{T}_{d}^{b} \\
\tau_{c / i}^{b}
\end{array}\right]=\mathbf{G}\left[\begin{array}{l}
\boldsymbol{Z} \\
\boldsymbol{\delta}_{e}
\end{array}\right]
$$

To produce a commanded thrust and torque, the minimumnorm pseudoinverse of eq. 29) is found. This method does not ensure that the control inputs will remain in a feasible range. Approaches to resolve this include saturating the results, limiting the aggressiveness of the trajectory, and using a linear program to find the minimum error solution [20]. In our simulation results, we saturated the control inputs prior to passing them into the simulation model.

\section{Simulation Results}

Simulation results for the controller developed here are shown in figs. 3 and 4 The vehicle simulated is the $0.77 \mathrm{~kg}$ E-flite Convergence tri-tilt-rotor. The full aerodynamic model and simulation environment are described in [17]. Knowledge of the full vehicle state was assumed in the simulation. Because this control scheme utilizes a more general trajectory than other controllers such as [14], we compare this controller to a controller developed for multirotors and typically used for eVTOLs in hover mode. The comparison controller assumes that the vehicle is only capable of producing thrust along $\boldsymbol{z}_{b}^{i}$; the desired attitude and thrust are determined using the method outlined in [1]. We refer to the multirotor-like controller as the $T_{x}=0$ controller and we refer to our proposed controller as the $\theta, \boldsymbol{T}$ optimized controller. Both controllers share the same attitude, rate, and control allocation components. The reference trajectory is a three-times differentiable polynomial spline, computed in a manner similar to that shown in [21]. The trajectory keypoints are chosen to create a typical urban air mobility scenario, with vertical takeoff, level flight up to moderate speeds of $6.4 \mathrm{~m} / \mathrm{s}$, and vertical landing while yawing $90^{\circ}$.
Figure 3 shows the simulated position trajectories for the two controllers. Both controllers experience some tracking error, with the majority being in the $z_{i}^{i}$ direction. We attribute these errors to the aerodynamics present in the simulation model that are not included in the optimization and control allocation models. Tracking errors are also caused by the saturation of control inputs calculated in the control allocation.

Figure 4 shows position error, thrust magnitude, and pitch angle for both controllers simulated on the same trajectory as shown in fig. 3. The average position error for the proposed $\theta$ and $\boldsymbol{T}$ optimized control method is $0.83 \mathrm{~m}$, and the average position error for the $T_{x}=0$ control method is $1.6 \mathrm{~m}$. The average thrust used by the proposed method is $8.86 \mathrm{~N}$, and is $10.06 \mathrm{~N}$ for the $T_{x}=0$ control method. The reduced thrust is due to the proposed method taking advantage of aerodynamic lift, even at low airspeeds. Notice that between 5 and 15 seconds, the thrust of the proposed control method drops to over half the thrust of the $T_{x}=0$ method. This occurs during the portion of flight where the vehicle travels at approximately $6 \mathrm{~ms}^{-1}$, a speed that falls in the transitioning range where partial lift is produced. In the lowest panel, the pitch angle of the proposed method can be seen to saturate at $\theta_{\max }$ for much of the trajectory. This allows it to generate maximum lift, reducing necessary additional thrust.

This comparison demonstrates how the energy consumption of a transitioning UAV in low-speed flight improves when its unique aerodynamic and control characteristics are explicitly considered in the control design.

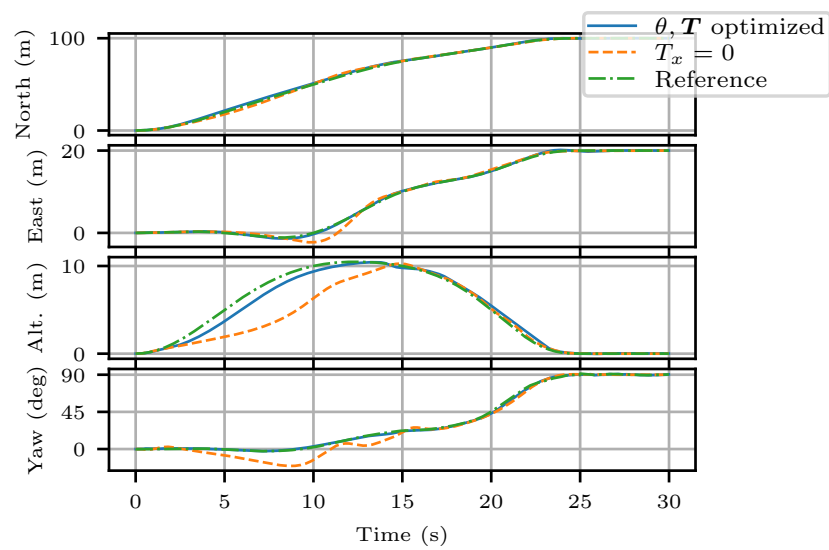

Fig. 3. Simulated position trajectories of the proposed $\theta$ and $\boldsymbol{T}$ optimized control method, compared with simulated trajectories of a geometric controller which assumes $T_{x}=0$, similar to that found in [1], shown along with the reference trajectory $\boldsymbol{p}_{r / i}^{i}$. The simulation environment and full simulation model are described in [17]. Both controllers experience some tracking error, with the majority being in the $\boldsymbol{z}_{i}^{i}$ direction. We attribute these errors to the aerodynamics present in the simulation model that are not included in the optimization and control allocation models.

\section{CONCLUSION AND FUTURE WORK}

We have developed a nonlinear trajectory tracking controller for winged eVTOL UAVs capable of producing thrust in the body longitudinal plane. To efficiently allocate thrust, we use a nonlinear optimization to find the maximum lift 


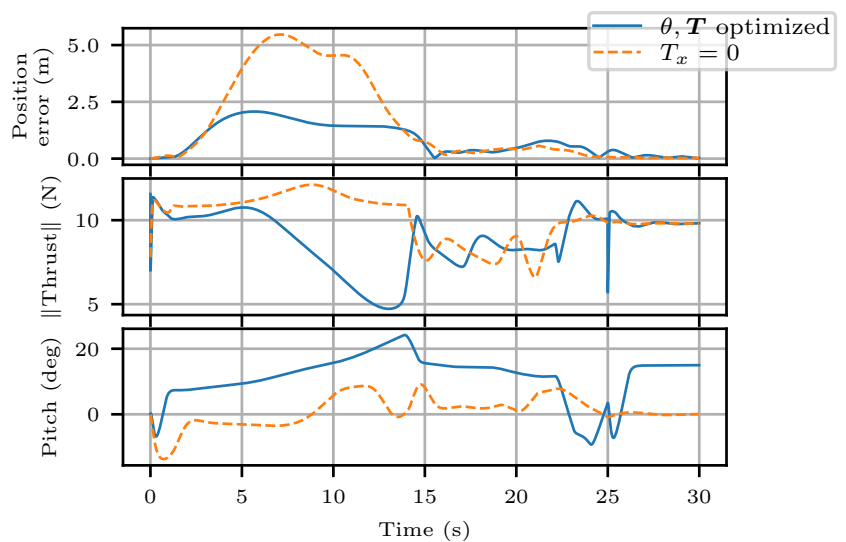

Fig. 4. Comparison of position error, thrust magnitude, and pitch angle for the same reference trajectory as is shown in fig. 3 The average position error for the proposed $\theta$ and $\boldsymbol{T}$ optimized control method is half the average position error for the $T_{x}=0$ control method. The average thrust used by the proposed method is $20 \%$ less than the $T_{x}=0$ control method. The reduced thrust is due to the proposed method taking advantage of aerodynamic lift, even at low airspeeds. In the lowest panel, the pitch angle of the proposed method can be seen to saturate at $\theta_{\max }$ for much of the trajectory. This allows it to generate maximum lift, reducing necessary additional thrust.

the vehicle can produce given desired flight conditions. To control the attitude of the vehicle we use an attitude controller developed on $\mathrm{SO}(3)$ and PID rate control. To find the actuator commands, we perform a linear control allocation given thrust and torque virtual inputs. Separating the attitude and rate control, and using a linear control allocation method allows for future implementation of our work on flight hardware.

The architecture we have proposed provides a novel method for tracking a larger set of trajectories than has previously been proposed. We believe this work can provide a framework for the development of robust trajectory tracking controllers for winged eVTOL UAVs. Future work includes developing new methods for modelling or estimating the aerodynamic characteristics of the vehicle. This, along with a reduction in computation time would improve the pitch and thrust optimization. Estimating disturbances, using an airspeed-dependent rotor model, and handling saturated input constraints would improve the control allocation.

\section{APPENDIX}

\section{A. Proof of Theorem 1}

Proof: We begin by assuming that the attitude and forces are achieved instantaneously, so $R_{b}^{i}=R_{d}^{i}$ and $\boldsymbol{F}_{b}^{b}=\boldsymbol{F}_{d}^{d}$, and we assume that

$$
R_{b}^{i} B \boldsymbol{F}_{b}^{b}=R_{d}^{i} B \boldsymbol{F}_{d}^{d}=\boldsymbol{f}_{d}^{i}
$$

Substituting eq. (30) in eq. (5) gives

$$
\begin{aligned}
& \ddot{\boldsymbol{p}}_{b / r}^{i}=g \boldsymbol{e}_{3}+\frac{1}{m} \boldsymbol{f}_{d}^{i}-\ddot{\boldsymbol{p}}_{r / i}^{i} \\
& =g \boldsymbol{e}_{3}+\frac{1}{m} m\left(\ddot{\boldsymbol{p}}_{r / i}^{i}-g \boldsymbol{e}_{3}-K_{p} \boldsymbol{p}_{b / r}^{i}-K_{d} \dot{\boldsymbol{p}}_{b / r}^{i}\right)-\ddot{\boldsymbol{p}}_{r / i}^{i} \\
& =-K_{p} \boldsymbol{p}_{b / r}^{i}-K_{d} \dot{\boldsymbol{p}}_{b / r}^{i}
\end{aligned}
$$

which, with $K_{p}, K_{d} \in \mathbb{R}^{3 \times 3}, K_{p}=K_{p}^{\top}>0$ and $K_{d}=$ $K_{d}^{\top}>0$, is a stable second-order system. To show that our assumption in eq. (30) holds, we first multiply the second equality on both sides by $\left(\boldsymbol{x}_{d}^{i}\right)^{\top},\left(\boldsymbol{y}_{d}^{i}\right)^{\top}$, and $\left(\boldsymbol{z}_{d}^{i}\right)^{\top}$, to obtain

$$
\begin{aligned}
F_{x, d} & =\left(\boldsymbol{x}_{d}^{i}\right)^{\top} \boldsymbol{f}_{d}^{i} \\
0 & =\left(\boldsymbol{y}_{d}^{i}\right)^{\top} \boldsymbol{f}_{d}^{i} \\
F_{z, d} & =\left(\boldsymbol{z}_{d}^{i}\right)^{\top} \boldsymbol{f}_{d}^{i}
\end{aligned}
$$

respectively. We now find $\boldsymbol{x}_{d}^{i}, \boldsymbol{y}_{d}^{i}$, and $\boldsymbol{z}_{d}^{i}$ so that these relationships hold, and such that $\psi_{d / i}=\psi_{r / i}$. Since we are assuming zero pitch, $\boldsymbol{x}_{d}^{i}$ will lie in the inertial horizontal plane and will be fully defined by $\psi_{r / i}$ :

$$
\boldsymbol{x}_{d}^{i}=\left[\cos \psi_{r / i}, \sin \psi_{r / i}, 0\right]^{\top} .
$$

To satisfy eq. 35 and the orthogonality of $R_{d}^{i}$ it is necessary to pick $\boldsymbol{y}_{d}^{i}$ such that it is orthogonal to both $\boldsymbol{f}_{d}^{i}$ and $\boldsymbol{x}_{d}^{i}$. To do so, we make the assumption that $\boldsymbol{x}_{d}^{i} \neq \boldsymbol{f}_{d}^{i}$. The same assumption is made in [1], and will only be violated if it is desired for the UAV to accelerate downwards at $g \frac{\mathrm{m}}{\mathrm{s}^{2}}$, which is unlikely and physically impossible due to the drag of the wing and the constraints on the orientation of the rotors. We then have

$$
\boldsymbol{y}_{d}^{i}=\frac{\boldsymbol{x}_{d}^{i} \times \boldsymbol{f}_{d}^{i}}{\left\|\boldsymbol{x}_{d}^{i} \times \boldsymbol{f}_{d}^{i}\right\|}
$$

By eliminating accelerations along $\boldsymbol{y}_{d}^{i}$, this additionally satisfies the coordinated flight condition for fixed-wing vehicles. Finally, $\boldsymbol{z}_{d}^{i}$ is chosen to be orthogonal to $\boldsymbol{x}_{d}^{i}$ and $\boldsymbol{y}_{d}^{i}$ and to satisfy the right hand rule

$$
\boldsymbol{z}_{d}^{i}=\boldsymbol{x}_{d}^{i} \times \boldsymbol{y}_{d}^{i}
$$

Therefore the choice of $R_{d}^{i}=\left[\boldsymbol{x}_{d}^{i}, \boldsymbol{y}_{d}^{i}, \boldsymbol{z}_{d}^{i}\right]$ satisfies eq. 30.

\section{B. Time Derivative of $R_{d}^{i}$}

The time derivative of $R_{d}^{i}$ can be found by differentiating $\boldsymbol{x}_{d}^{i}, \boldsymbol{y}_{d}^{i}$, and $\boldsymbol{z}_{d}^{i}$,

$$
\dot{R}_{d}^{i}=\left[\dot{\boldsymbol{x}}_{d}^{i}, \dot{\boldsymbol{y}}_{d}^{i}, \dot{\boldsymbol{z}}_{d}^{i}\right]
$$

These derivatives are

$$
\begin{aligned}
\dot{\boldsymbol{x}}_{d}^{i}= & {\left[-\dot{\psi}_{r / i} \sin \psi_{r / i}, \dot{\psi}_{r / i} \cos \psi_{r / i}, 0\right]^{\top}, } \\
\dot{\boldsymbol{y}}_{d}^{i}= & \frac{\dot{\boldsymbol{x}}_{d}^{i} \times \boldsymbol{f}_{d}^{i}+\boldsymbol{x}_{d}^{i} \times \dot{\boldsymbol{f}}_{d}^{i}}{\left\|\boldsymbol{y}_{d}^{i}\right\|} \\
& -\boldsymbol{y}_{d}^{i} \frac{\left(\boldsymbol{y}_{d}^{i}\right)^{\top}\left(\dot{\boldsymbol{x}}_{d}^{i} \times \boldsymbol{f}_{d}^{i}+\boldsymbol{x}_{d}^{i} \times \dot{\boldsymbol{f}}_{d}^{i}\right)}{\left\|\boldsymbol{y}_{d}^{i}\right\|^{3}}
\end{aligned}
$$




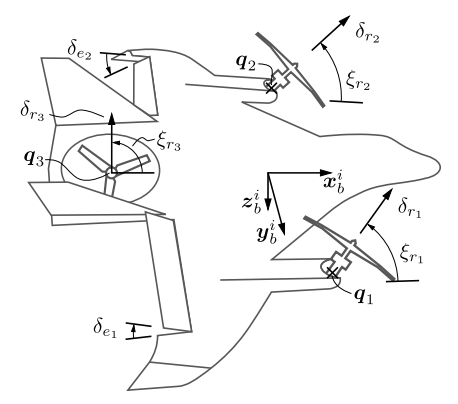

Fig. 5. Notation used for the actuators on the E-flite Convergence tri-tiltrotor eVTOL. Actuators include the rotor throttle $\delta_{r_{*}}$, rotor angle $\xi_{e_{*}}$, and elevon deflection $\delta_{e_{*}}$. Also shown are the rotor positions, denoted $\boldsymbol{q}_{*}$.

with

$$
\begin{aligned}
\dot{\boldsymbol{f}}_{d} & =\frac{d}{d t} m\left(\ddot{\boldsymbol{p}}_{r / i}^{i}-g \boldsymbol{e}_{3}-K_{p} \boldsymbol{p}_{b / r}^{i}-K_{d} \dot{\boldsymbol{p}}_{b / r}^{i}\right) \\
& =m\left(\dddot{\boldsymbol{p}}_{r / i}^{i}-K_{p} \dot{\boldsymbol{p}}_{b / r}^{i}-K_{d} \ddot{\boldsymbol{p}}_{b / r}^{i}\right) \\
& =m\left(\dddot{\boldsymbol{p}}_{r / i}^{i}-K_{p} \dot{\boldsymbol{p}}_{b / r}^{i}-K_{d}\left(-K_{p} \boldsymbol{p}_{b / r}^{i}-K_{d} \dot{\boldsymbol{p}}_{b / r}^{i}\right)\right)
\end{aligned}
$$

and finally,

$$
\dot{\boldsymbol{z}}_{d}^{i}=\dot{\boldsymbol{x}}_{d}^{i} \times \boldsymbol{y}_{d}^{i}+\boldsymbol{x}_{d}^{i} \times \dot{\boldsymbol{y}}_{d}^{i}
$$

\section{Control Allocation}

Here the goal is to transform the desired thrust $\boldsymbol{T}_{d}^{b}=$ $\left[T_{x, d}^{b}, T_{z, d}^{b}\right]$ and the desired torque $\tau_{c / b}^{b}=\left[\tau_{x}, \tau_{y}, \tau_{z}\right]$ to individual motor throttle, motor angle, and control surface commands. The vehicle we consider is the tri-tilt-rotor Convergence aircraft manufactured by E-flite. A diagram showing the naming conventions for the vehicle actuators is shown in fig. 5. The actuator inputs are rotor throttles, $\boldsymbol{\delta}_{r}=\left[\delta_{r_{1}}, \delta_{r_{2}}, \delta_{r_{3}}\right]$ where $\delta_{r_{*}} \in[0,1]$, rotor angles $\boldsymbol{\xi}_{r}=$ $\left[\xi_{r_{1}}, \xi_{r_{2}}, \xi_{r_{3}}\right]$ where $\xi_{r_{*}} \in\left[\xi_{\min }, \xi_{\text {max }}\right]$ with $\xi_{r_{3}}=\frac{\pi}{2}$, and the elevon deflections $\boldsymbol{\delta}_{e}=\left[\delta_{e_{1}}, \delta_{e_{2}}\right]$ where $\delta_{e_{*}} \in[-1,1]$. The model is described in more detail in [17].

We begin by finding a linear relationship between the rotor command $\delta_{r_{*}}$ and the thrust and torque produced by that rotor. We then assemble an allocation matrix using the geometry of the vehicle and augment it with an airspeed dependent elevon model.

1) Linear Rotor Model: The airspeed of a multirotor is always near zero, so it is typical to use a linear rotor model for both thrust and torque [3]. As the airspeed of the vehicle increases, it has a significant affect on the performance of the propeller. While this affect occurs, the use of feedback control can allow the control allocation scheme to use a linear model which assumes zero airspeed. In future work, a more precise approach will be to vary the linear model based on the current airspeed of the vehicle.

Define $\boldsymbol{q}_{i}$ to be the measured rotor positions and define the rotor axis direction vector to be

$$
\boldsymbol{s}_{r_{i}}=\left[\cos \xi_{r_{i}}, 0,-\sin \xi_{r_{i}}\right]^{\top} .
$$

At hover, the rotor thrust and torque is approximately linear in $\boldsymbol{\delta}_{r}$, and we denote the linear coefficients as $k_{T_{i}} \in \mathbb{R}$ for the thrust, and $k_{M_{i}} \in \mathbb{R}$ for the torque. These coefficients can be found experimentally for a vehicle in hover by measuring the attitude $R_{b}^{i}$, commanded throttles $\delta_{r}$, rotor angles $\boldsymbol{\xi}_{r}$, and assuming the rotor positions are known. Since the aerodynamic forces and moments are zero when in hover, we have the force balance

$$
\begin{aligned}
R_{i}^{b} \boldsymbol{F}_{\text {hover }} & =\sum_{i=1}^{3} k_{T_{i}} \delta_{r_{i}} \boldsymbol{s}_{r_{i}} \\
\boldsymbol{M}_{\text {hover }} & =\sum_{i=1}^{3} k_{T_{i}} \delta_{r_{i}}\left(\boldsymbol{q}_{i} \times \boldsymbol{s}_{r_{i}}\right)-k_{M_{i}} \delta_{r_{i}} \boldsymbol{s}_{r_{i}}
\end{aligned}
$$

where $\boldsymbol{F}_{\text {hover }}=[0,0,-m g]^{\top}$ and $\boldsymbol{M}_{\text {hover }}=[0,0,0]^{\top}$. Since $\boldsymbol{s}_{r_{i}}^{\top} \boldsymbol{e}_{2}=0$, this produces a system of 5 equations and 6 unknowns. The front two rotors use identical motors, so the two additional constraints $k_{T_{1}}=k_{T_{2}}$ and $k_{M_{1}}=k_{M_{2}}$ are used to make the system overconstrained. The least-squares solution is then used to find $k_{T_{i}}$ and $k_{M_{i}}$.

2) Allocation Matrix: Once $k_{T_{i}}$ and $k_{M_{i}}$ are determined, they can be used to construct a linear relationship from $\boldsymbol{\delta}_{r}$ and $\boldsymbol{\delta}_{e}$ to $\boldsymbol{T}_{d}^{b}$ and $\boldsymbol{\tau}_{c / i}^{b}$. To do so, we first note that

$$
\boldsymbol{q}_{i} \times \boldsymbol{s}_{r_{i}}=\left[\begin{array}{c}
-q_{i 2} \sin \left(\xi_{r_{i}}\right) \\
q_{i 3} \cos \left(\xi_{r_{i}}\right)+q_{i 1} \sin \left(\xi_{r_{i}}\right) \\
-q_{i 2} \cos \left(\xi_{r_{i}}\right)
\end{array}\right] .
$$

We then have

$$
\boldsymbol{T}=\sum_{i=1}^{3} k_{T_{i}} \delta_{i} \boldsymbol{s}_{r_{i}}
$$

and

$$
\boldsymbol{\tau}=\sum_{i=1}^{3} k_{T_{i}} \delta_{r_{i}}\left(\boldsymbol{q}_{i} \times \boldsymbol{s}_{r_{i}}\right)-k_{M_{i}} \delta_{r_{i}} \boldsymbol{s}_{r_{i}} .
$$

Due to the rotor angles, the relationships in eqs. (50) and (51) are not linear in the control variables. However, we can use a rectangular coordinate transform to define a new set of controls representing the body frame horizontal and vertical throttle commands,

$$
\begin{aligned}
& \zeta_{i x}=\delta_{r_{i}} \cos \left(\xi_{r_{i}}\right) \\
& \zeta_{i z}=\delta_{r_{i}} \sin \left(\xi_{r_{i}}\right)
\end{aligned}
$$

In the reverse, we have,

$$
\delta_{r_{i}}=\sqrt{\zeta_{i x}^{2}+\zeta_{i z}^{2}}
$$

and

$$
\xi_{r_{i}}=\tan ^{-1}\left(\frac{\zeta_{1 z}}{\zeta_{1 x}}\right) .
$$

The second row of eq. 50] is all zeros, and $\zeta_{3 x}=0$. Define

$$
\boldsymbol{Z}=\left[\zeta_{1 x}, \zeta_{1 z}, \zeta_{2 x}, \zeta_{2 z}, \zeta_{3 z}\right]
$$

then, the matrix form of eqs. 50 and (51) can be written as

$$
\left[\begin{array}{c}
\boldsymbol{T}_{b}^{b} \\
\tau_{b / i}^{b}
\end{array}\right]=\mathbf{G}_{r} \boldsymbol{Z}
$$


TABLE I

ACTUATOR TO VIRTUAL INPUT MATRIX RELATIONSHIP

$$
\left[\begin{array}{c}
\boldsymbol{T}_{d}^{b} \\
\tau_{c / i}^{b}
\end{array}\right]=\mathbf{G}\left[\begin{array}{c}
\boldsymbol{Z} \\
\boldsymbol{\delta}_{e}
\end{array}\right]=\left[\begin{array}{cccccccc}
k_{T_{1}} & 0 & k_{T_{2}} & 0 & k_{T_{3}} & 0 & 0 & 0 \\
0 & -k_{T_{1}} & 0 & -k_{T_{2}} & 0 & -k_{T_{3}} & 0 & 0 \\
-k_{M_{1}} & -k_{T_{1}} q_{12} & -k_{M_{2}} & -k_{T_{1}} q_{22} & -k_{M_{3}} & -k_{T_{3}} q_{32} & \Gamma C_{l_{\delta_{e}}} & \Gamma C_{l_{\delta_{e}}} \\
k_{T_{1}} q_{13} & k_{T_{1}} q_{11} & k_{T_{2}} q_{23} & k_{T_{2}} q_{21} & k_{T_{3}} q_{33} & k_{T_{3}} q_{31} & -\Gamma C_{m_{\delta_{e}}} & \Gamma C_{m_{\delta_{e}}} \\
-k_{T_{1}} q_{12} & k_{M_{1}} & -k_{T_{2}} q_{22} & k_{M_{2}} & -k_{T_{3}} q_{32} & k_{M_{3}} & 0 & 0
\end{array}\right]\left[\begin{array}{c}
\zeta_{1 x} \\
\zeta_{1 z} \\
\zeta_{2 x} \\
\zeta_{2 z} \\
\zeta_{3 x} \\
\zeta_{3 z} \\
\delta_{e_{1}} \\
\delta_{e_{2}}
\end{array}\right]
$$

To perform control allocation for hover flight, eq. (57) can be inverted. However, this does not include the relationships necessary to allocate the use of the elevons in fixed wing flight. A linear model for the moments produced by an elevon is

$$
M_{\delta_{e}}=\frac{1}{2} \rho V_{a}^{2} S b C_{*_{e}} \delta_{e}
$$

where $C_{* \delta_{e}}=C_{l_{\delta_{e}}}$ for the rolling moment and where $C_{* \delta_{e}}=$ $C_{m_{\delta_{e}}}$ for the pitching moment. The moment produced by an elevon is proportional to the square of $V_{a}$. We use the measured airspeed to determine how effective the elevons will be at the current flight condition. We then augment $\mathbf{G}_{r}$ with the elevon torques to represent the relationship between all available actuators and the thrust and torques they produce. Defining $\Gamma\left(V_{a}\right)=\frac{1}{2} \rho V_{a}^{2} S b$, and $\mathbf{G}=\left[\mathbf{G}_{r}, \mathbf{G}_{e}\right]$, where $\mathbf{G}_{e}$ is the elevon allocation matrix, we have the result shown in table I]

\section{REFERENCES}

[1] T. Lee, M. Leok, and N. H. McClamroch, "Geometric tracking control of a quadrotor UAV on SE(3)," in 49th IEEE Conference on Decision and Control (CDC), 2010, pp. 5420-5425.

[2] A. Bry, C. Richter, A. Bachrach, and N. Roy, "Aggressive flight of fixed-wing and quadrotor aircraft in dense indoor environments," The International Journal of Robotics Research, vol. 34, no. 7, pp. 9691002, 2015.

[3] R. Mahony, V. Kumar, and P. Corke, "Multirotor aerial vehicles: Modeling, estimation, and control of quadrotor," IEEE Robotics and Automation magazine, vol. 19, no. 3, pp. 20-32, 2012.

[4] D. Mellinger, N. Michael, and V. Kumar, "Trajectory generation and control for precise aggressive maneuvers with quadrotors," The International Journal of Robotics Research, vol. 31, no. 5, pp. 664674, 2012.

[5] M. Costandin, B. Costandin, and P. Dobra, "Nonlinear model and trajectory control of a novel VTOL vehicle II," in 2018 International Conference on Unmanned Aircraft Systems (ICUAS), June 2018, pp. 806-815.

[6] C. Papachristos, K. Alexis, and A. Tzes, "Dual-authority thrustvectoring of a tri-tiltrotor employing model predictive control," Journal of intelligent \& robotic systems, vol. 81, no. 3-4, pp. 471-504, 2016.
[7] A. S. Saeed, A. B. Younes, S. Islam, J. Dias, L. Seneviratne, and G. Cai, "A review on the platform design, dynamic modeling and control of hybrid UAVs," in 2015 International Conference on Unmanned Aircraft Systems (ICUAS). IEEE, 2015, pp. 806-815.

[8] P. Morin, "Modeling and control of convertible micro air vehicles," in 2015 10th International Workshop on Robot Motion and Control (RoMoCo), 2015, pp. 188-198.

[9] Z. Liu, Y. He, L. Yang, and J. Han, "Control techniques of tilt rotor unmanned aerial vehicle systems: A review," Chinese Journal of Aeronautics, vol. 30, no. 1, pp. 135-148, 2017.

[10] G. Di Francesco, E. D'Amato, and M. Mattei, "INDI control with direct lift for a tilt rotor UAV," IFAC-PapersOnLine, vol. 48, no. 9, pp. 156-161, 2015.

[11] L. Meier, D. Honegger, and M. Pollefeys, "PX4: A node-based multithreaded open source robotics framework for deeply embedded platforms," in 2015 IEEE international conference on robotics and automation (ICRA). IEEE, 2015, pp. 6235-6240.

[12] G. Notarstefano and J. Hauser, "Modeling and dynamic exploration of a tilt-rotor VTOL aircraft," IFAC Proceedings Volumes, vol. 43, no. 14, pp. 119-124, 2010.

[13] G. Flores and R. Lozano, "Transition flight control of the quadtilting rotor convertible MAV," in 2013 International Conference on Unmanned Aircraft Systems (ICUAS), 2013, pp. 789-794.

[14] A. Anglade, J. Kai, T. Hamel, and C. Samson, "Automatic control of convertible fixed-wing drones with vectorized thrust," in 2019 IEEE 58th Conference on Decision and Control (CDC), 2019, pp. 58805887.

[15] T. Airimitoaie, G. P. Aguilar, L. Lavigne, C. Farges, and F. Cazaurang, "Convertible aircraft dynamic modelling and flatness analysis," IFACPapersOnLine, vol. 51, no. 2, pp. 25-30, 2018.

[16] P. Hartmann, C. Meyer, and D. Moormann, "Unified velocity control and flight state transition of unmanned tilt-wing aircraft," Journal of guidance, control, and dynamics, vol. 40, no. 6, pp. 1348-1359, 2017.

[17] J. Willis, J. Johnson, and R. W. Beard, "State-dependent LQR control for a tilt-rotor UAV," in 2020 American Control Conference (ACC), 2020, pp. 4175-4181.

[18] P. E. Gill, W. Murray, and M. A. Saunders, "SNOPT: An SQP Algorithm for Large-scale Constrained Optimization," SIAM review, vol. 47, no. 1, pp. 99-131, 2005.

[19] R. E. Perez, P. W. Jansen, and J. R. R. A. Martins, "pyOpt: A Python-based object-oriented framework for nonlinear constrained optimization," Structural and Multidisciplinary Optimization, vol. 45, no. 1, pp. 101-118, January 2012.

[20] T. A. Johansen and T. I. Fossen, "Control allocation-a survey," Automatica, vol. 49, no. 5, pp. 1087-1103, 2013.

[21] C. Richter, A. Bry, and N. Roy, "Polynomial trajectory planning for aggressive quadrotor flight in dense indoor environments," in Robotics Research. Springer, 2016, pp. 649-666. 